DOI: $10.1515 /$ awutp -2015-0205

\title{
NONTHERMAL ARGON PLASMA GENERATOR AND SOME POTENTIAL APPLICATIONS
}

\author{
M. Bunoiu ${ }^{1}$, I. Jugunaru ${ }^{1}$, I. Bica ${ }^{1}$, M. Balasoiu ${ }^{2,3^{*}}$ \\ ${ }^{I}$ Faculty of Physics, West University of Timisoara, 300223-Timisoara \\ ${ }^{2} J o i n t$ Institute for Nuclear Research, Dubna, 141980 Moscow Region, Russian Federation \\ ${ }^{3}$ Horia Hulubei National Institute for Physics and Nuclear Engineering, Bucharest, Romania \\ *Corresponding author: balas@jinr.ru
}

Article Info

Received: 22.09 .2015

Accepted: 25.11.2015

Keywords: nonthermal plasma, tungsten electrode, nozzle, fiber, blood material, human hair.

\begin{abstract}
A laboratory - made nonthermal plasma generator is presented. It has a diameter of $0.020 \mathrm{~m}$ and length of $\quad 0.155 \mathrm{~m}$ and contains two electrodes. The first electrode is a $2 \%$ Th-W alloy, $0.002 \mathrm{~m}$ in diameter bar, centred inside the generator's body by means of a four channel teflon piece; the other three channels, $0.003 \mathrm{~m}$ in diameter, are used for Ar supply. The second electrode is a nozzle of $0.002 \mathrm{~m}-0.008 \mathrm{~m}$ diameter and $0.005 \mathrm{~m}$ length. A $\sim 500 \mathrm{kV} / \mathrm{m}$ electric field is generated between the two electrodes by a high frequency source (13.56 $\mathrm{MHz} \pm 5 \%$ ), equipped with a OT-1000 (Tungsram) power triode. For Ar flows ranging from $0.00008 \mathrm{~m}^{3} / \mathrm{s}$ to $0.00056 \mathrm{~m}^{3} / \mathrm{s}$, a plasma jet of length not exceeding $0.015 \mathrm{~m}$ and temperature below $315 \mathrm{~K}$ is obtained. Anthurium andraeanumis sample, blood matrix, human hair and textile fibers may be introduced in the plasma jet. For time periods of $30 \mathrm{~s}$ and $60 \mathrm{~s}$, various effects like, cell detexturization, fast blood coagulation or textile fiber or hair cleaning and smoothing are obtained. These effects are presented and discussed in the paper.
\end{abstract}

\section{Introduction}

The plasma obtained by electric discharge can be either thermal, when the temperature of the electrons $\left(T_{e}\right)$ is small compared to that of the ions or atoms $\left(T_{i}\right)$, or non-thermal when $T_{e} \gg T_{i}[1]$. Both types of plasma are of interest for various applications. Thus, thermal plasma can be used for highly alloyed steels cutting and welding [2], tough and extra-tough semi-products splintering [2, 3-5], or fine and ultra-fine powders production [2, 4, 5]. On the other hand, the non-thermal plasma can be used for biological environment decontamination [6-9], biocompatible surfaces preparation [10], cancer therapy [11], gum and dental cavities 
treatment [12], etc. The interest in nonthermal plasma has significantly increased during the last few years [7-17]. In all these applications (like in many others), an important role is played by the plasma generator; the present article proposes a non-thermal plasma generator as well as some results obtained in processing vegetal, biological and non-biological materials.

\section{Description}

The schematic drawing of the non-thermal plasma generator (NPG) is presented in Fig. 1. It contains a nozzle subassembly and an electrode subassembly, mounted and positioned inside a common body. The nozzle subassembly contains the nozzle 1 and the nozzle support 2. Both are made from cooper alloy. They are fixed inside the ceramic body 3 , thus forming a compact body. The nozzle 1 is interchangeable. The diameter of the nozzle $\left(\phi_{\mathrm{i}}\right)$ can take values up to $0.01 \mathrm{~m}$. The electrode subassembly contains the body 4 , made from teflon, and the tungsten electrode (position 5 in Fig.1). The role of the body (pos. 4) is to secure and position the tungsten electrode. Along its length three channels are designed, which communicate with the gas admission nozzle (pos. 9 in Fig. 1) and the pressure equalizing chamber. Each channel (pos. 11 in Figure 1) has a diameter of $0.003 \mathrm{~m}$. The angular distance between the channels is $120^{\circ}$. The two subassemblies are fixed with the nut 6 in the terminal 8 , both from teflon. The power supply of the NPG is done through the terminal 9 and nozzle 1. The plasma gas (Ar) of the NPG is supplied from a 150 bar compressed gas cylinder through a pressure reducer and controlled by means of a gas flow-meter. At the generator input (pos. 9 in Fig. 1), the Ar flow is preset to a constant value.

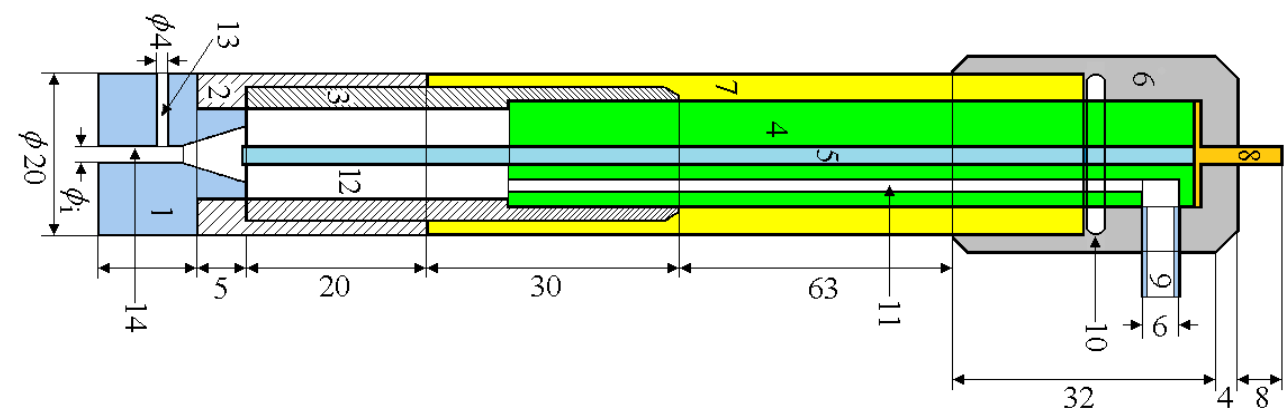

Fig. 1 Nonthermal plasma generator (ensemble drawing): 1, interchangeable nozzle; 2, nozzle support; 3 , ceramic body; 4, mounting body; 5, tungsten electrode; 6 , nut; 7, collar sheath; 8 , terminal; 9 , Ar inlet tube; 10 , sealing ring; 11, gas admission channel; 12, pressure equalizing chamber; 13, transporting gas admission channel; 14 , nozzle channel; $\phi_{\mathrm{i}}$, nozzle diameter. 
The NPG's nozzle is provided with a transporting gas admission channel (pos. 13 in Fig. 1), through which materials like aerosols, are introduced in the nozzle channel (pos. 13 in Fig. 1).

\section{Nonthermal plasma generation}

In Figure 2 the ensemble configuration of the installation for non-thermal Ar plasma generation is presented. The installation contains a high frequency generator (IFP), a tuning capacitor $C$, the plasma generator $(N P G)$ and the compressed gas reservoir $(A r) . I F P$ is an oscillator using a power vacuum triode (OT-1000, Tungsram). The maximum power transferred to the electric discharge through $a$ and $b$ terminals, is $80 \mathrm{~W}$. at $\sim 1000 \mathrm{~V}_{\text {ac }}$ The IFP working frequency is $13.56 \mathrm{MHz}( \pm 5 \%)$. The capacitor $C$ (used for load tunning [15]) can be continuously trimmed between $17 p F$ and $245 p F$. By adjusting $C$, continuously adjustable transfer can be achieved, from the IFP output to the capacitive discharge. The tungsten electrode 5 of the plasma generator, is connected to terminal $a$ of the IFP, while the nozzle is connected to the ground. The distance between the tungsten electrode and the nozzle is set to $0.004 \mathrm{~m}$. The Ar reservoir contains a gas tank (200 bar) and a pressure reductor (200 bar $/ 1.5$ bar), provided with a flow-meter. The gas flow can be adjusted between $g=0.00004 \mathrm{~m}^{3} / \mathrm{s}$ and $g=0.00056 \mathrm{~m}^{3} / \mathrm{s}$. Nozzles with different diameters may be mounted in the nozzle holder. For nozzles with the same diameter and fixed values of the Ar flow, the plasma jet is examined; several examples are shown in Figs. 3 and 4.

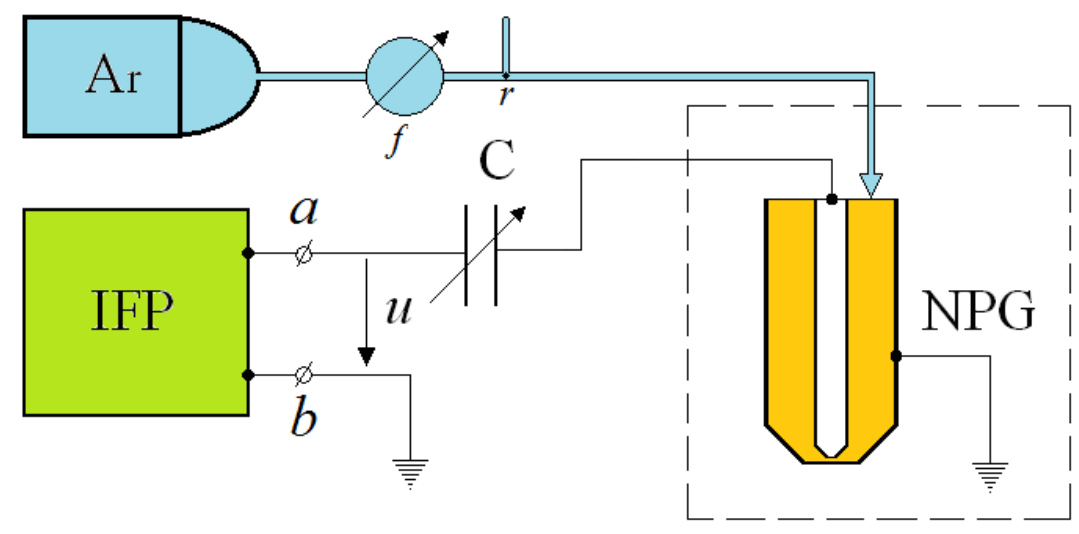

Fig. 2 Installation for nonthermal plasma generation (block diagram): Ar, compressed gas reservoir; $f$, flowmeter, $r$, pressure reductor, $I F P$, high frequency source; $C$, tuning capacitor; $N P G$, nonthermal plasma generator; $u$, high frequency voltage. 
Accordingly, by increasing the value of $C$, the phase lag between the current and the voltage decreases and, thus, the power transfer from IFP to $N P G$, is enhanced. The result is an increase of $n$ and, thus, as Fig.3 confirms, an increase of the plasma volume. Needle-shaped plasma is obtained when decreasing the diameter of the nozzle, from $0.010 \mathrm{~m}$ to $0.002 \mathrm{~m}$ (Fig.4b). For $C=20 p F$ a discharge is initiated at the top of the central electrode (Fig.3a). This discharge has the shape of a liquid drop.

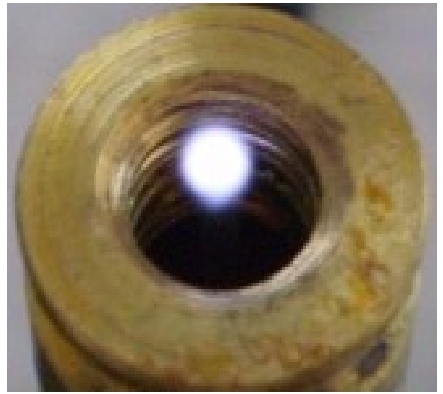

a)

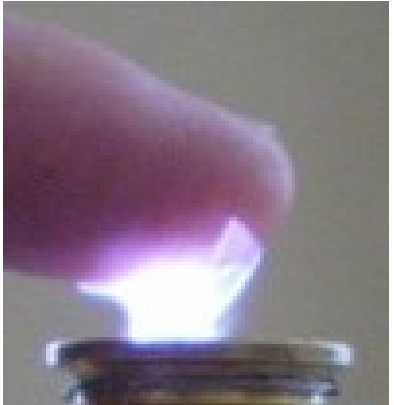

b)

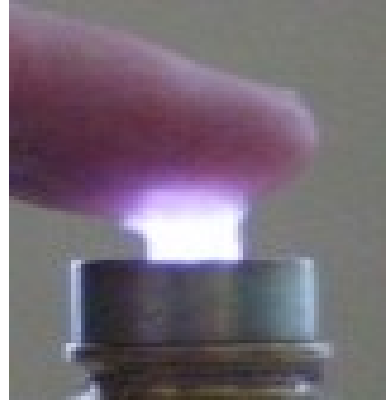

c)

Fig. 3 Nonthermal Ar plasma jet through a nozzle with a diameter of $0.010 \mathrm{~m}$, for different values of the tuning capacitor and of the Ar flow: a) $\mathrm{C}=20 \mathrm{pF}, g=0.00033 \mathrm{~m}^{3} / \mathrm{s}$; b) $\mathrm{C}=240 \mathrm{pF}$ and $g=0.00033 \mathrm{~m}^{3} / \mathrm{s}$, c) $\mathrm{C}=240 \mathrm{pF}$ and $g=0.00040 \mathrm{~m}^{3} / \mathrm{s}$.

By increasing $C$ at constant Ar flow, the plasma volume increases, and the drop-like discharge turns into a jet when $C=240 p F$ (Fig. $3 \mathrm{~b}$ and 3.c); when the Ar flow is increased to the value $g=0.00040 \mathrm{~m}^{3} / \mathrm{s}$, the diameter of the discharge becomes identical to that of the nozzle, of length now increased by $10 \mathrm{~mm}$ (Fig.3c). Using the plasma generator as described above, experiments were conducted on exposing glass and some organic materials to the plasma jet. By means of the infrared thermometer AX-6520 type (AXIO MET) the temperature on the surface of the observer's finger (Fig.3b and c, Fig. $4 b$ and c) is determined. All the measurements have indicated values below $315 \mathrm{~K}$.

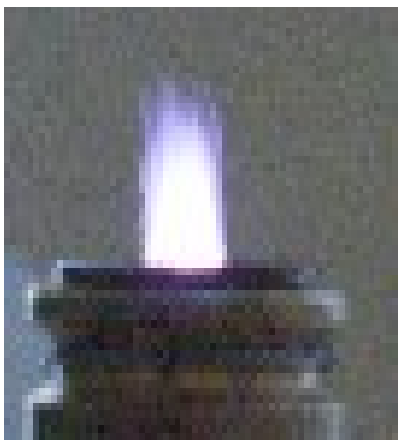

a)

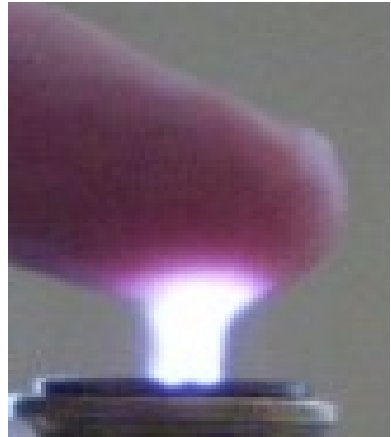

b)

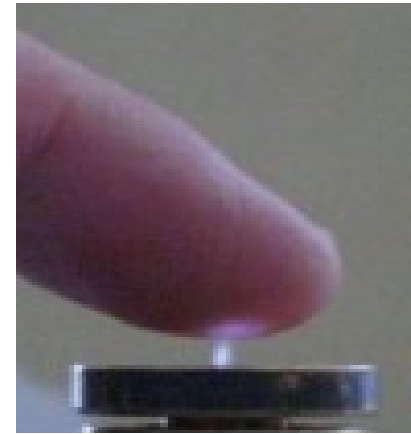

c)

Fig. 4 Nonthermal Ar plasma at $g=0.00033 \mathrm{~m}^{3} / \mathrm{s}$, for different nozzle diameters: a) $d=0.010 \mathrm{~m}$, b) $d=0.005 \mathrm{~m}$, and c) $d=0.002 \mathrm{~m}$. 


\section{Materials processing in nonthermal plasma}

The following values: $C=240 \mathrm{pF}, d=0.010 \mathrm{~m}$ and $g=0.00033 \mathrm{~m}^{3} / \mathrm{s}$ of the $N P G$ parameters were used during the experiments, while the distance from the nozzle to the exposed surface was maintained at $0.005 \mathrm{~m}$.

\subsection{Vegetal material}

A $20 \mathrm{~mm} \times 10 \mathrm{~mm} \times 2 \mathrm{~mm}$ sample was cut from a leaf of Anthurium andraeanumis and fixed on a microscope lamella and then examined by means of a BX51(Olympus) microscope both before and after being exposed to the plasma jet.

Micropscope image (Fig.5a) shows that the leaf surface is formed from cellular bulbs. Due to the action of the nonthermal plasma chemical reactions take place between bulbs and the absorbed air jet. Consequently, on the bulb's surface oxides occur in the form of films [15] as shown in Figs. 5b and 5c.

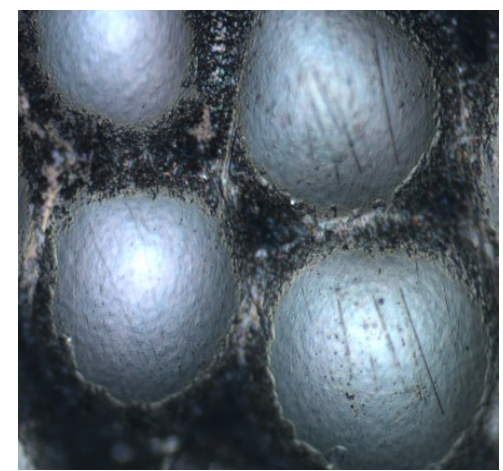

a)

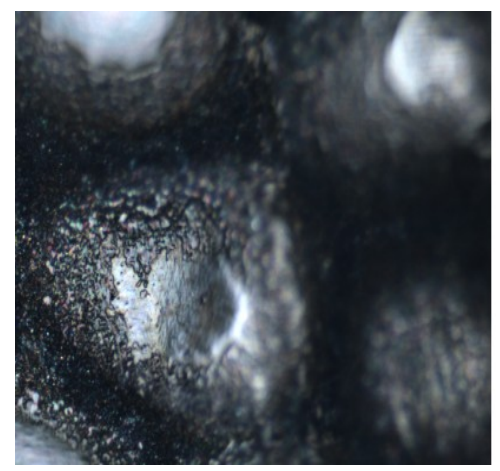

b)

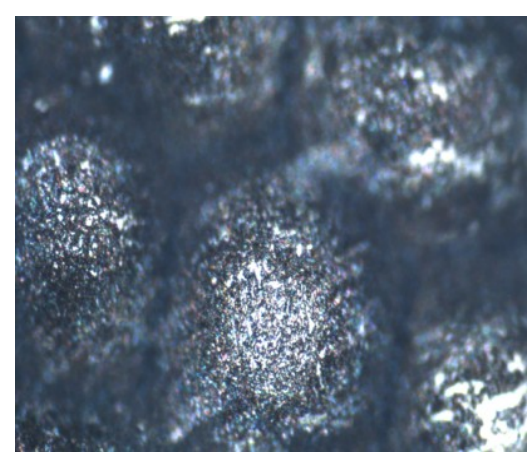

c)

Fig. 5. Foliage of Anthurium andraeanumis viewed with the optic microscope (magnification x20) unexposed in the plasma jet (a) and exposed in the plasma jet for $30 \mathrm{~s} \mathrm{(b)} \mathrm{and} 60 \mathrm{~s}$ respectively (c).

The ionized particles from the plasma interact with the cellular aggregates from the sample, causing dehydration. In addition, the ultraviolet radiation from the plasma discharge [16] leads to the formation of a residual shell, progressively covering the as-dehydrated aggregates. In Fig.5 the obtained results are shown.

\subsection{Blood material}

The blood material is deposited onto a microscope lamella (Fig.6a). In the microscope Olympus BX 51 it is viewed before and after the treatment in the argon plasma jet (Fig. 6b and c). It can be noticed from Fig.6, that under the action of the plasma for 30s (Fig.6 b) and for 60s (Fig.6c), the sample changes gradually the color. At the end of the $60 \mathrm{~s}$ time of 
exposure in the argon plasma jet, the blood sample presents itself as well-contoured formations (Fig. 6c). From these observations, it can be drown the conclusion that under the action of the plasma, the coagulation of the blood material is fast compared to clotting in the usual environment, known to be of 8-12 $\min$ [14].

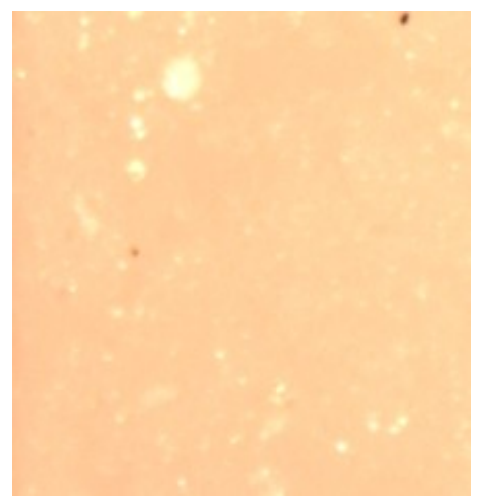

a)

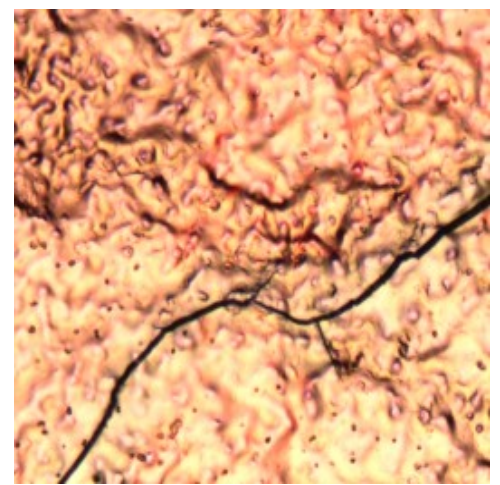

b)

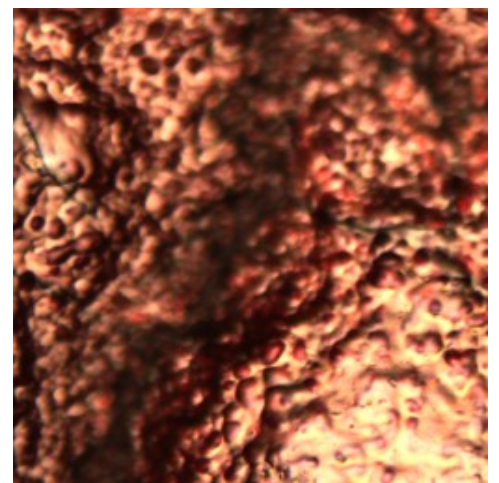

c)

Fig. 6. Blood material viewed at the optic microscope (magnification $\mathrm{x} 20$ ), before exposure in the argon plasma jet (a) and after argon plasma jet exposure, for a period of $30 \mathrm{~s}$ (b) and for a period of $60 \mathrm{~s}$, respectively (c).

Under the influence of plasma jet the ionization of blood constituents gets involved. Note that the related ions working in conjunction with double ionized Ca-ions play an important role in the blood coagulation, as explained in Ref. [18].

\subsection{Human hair}

A human hair fixed on a microscope lamella was exposed to the plasma jet to investigate the influence of the nonthermal plasma on the hair sheath. The hair is viewed with the microscope Olympus BX 51. The not treated in the plasma hair shaft is presented in Fig. 7a. It is knowm that on the surface of the hair a protective substance called creatine exists. In plasma, the thread does not change shape (Figs. 7 b, and c). That means that at the thermal plasma temperature chemical reactions that lead to observable effects on the surface of the hair do not occur [22]. 


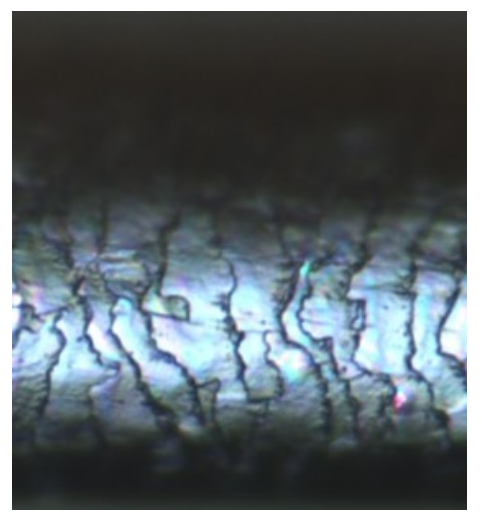

a)

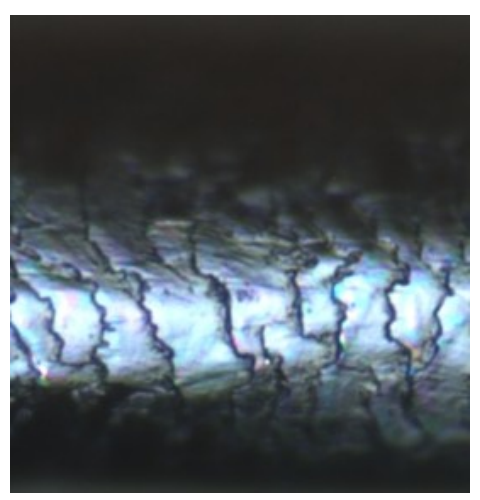

b)

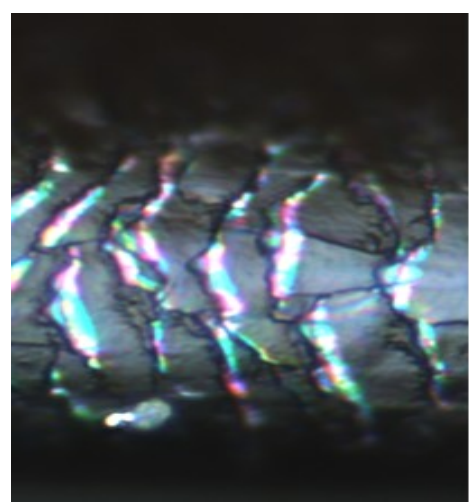

c)

Fig. 7. Views at the optic microscope (magnification 50x) of a human hair before the plasma exposure (a), after the plasma exposure for $60 \mathrm{~s}(\mathrm{~b})$ and $420 \mathrm{~s}$ respectively (c).

\subsection{Textile material}

Wires are pulled from a cloth that contains 30\% nylon and $70 \%$ cotton. A wire is fixed on a microscope lamella and it is observed with the microscope Olympus BX 51 before and after the exposure in the argon non-thermal plasma.

The wire appears as a wisp from which branches grow (Fig. 8a).Under the plasma jet, cloth-wires get increasingly smoothed (Fig.8b). In addition, studies done in Refs. [19, 20] show that the wire surface becomes sterilized. A textile yarn interaction with non-thermal plasma surface tension change occurs, increasing at the same time, the watering energy. Thus, textile fibers obtained from non-thermal plasma treatment turns in materials that do not get dirty.

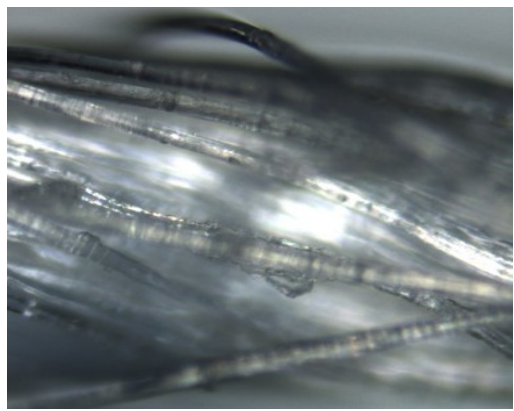

a)

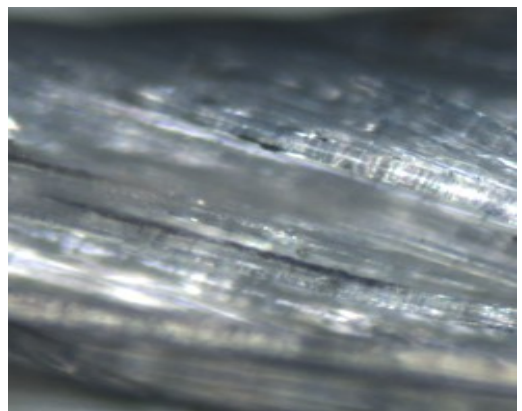

b)

Fig. 8. Cloth wire viewed at the optic microscope (magnification x20) before being plasma-exposed (a) and after the plasma exposure for a period of $60 \mathrm{~s}(\mathrm{~b})$. 


\subsection{The glass blade}

The glass blade is introduced in the plasma jet during approximately $300 \mathrm{~s}$. A petroleum drop is then deposited on the glass surface (Fig.9a).

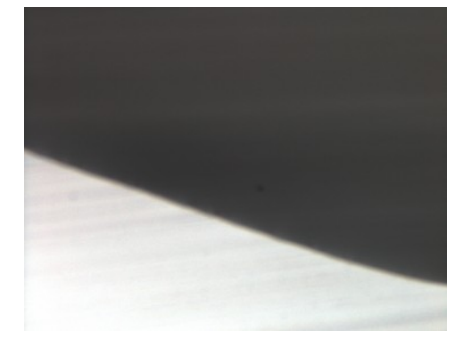

a)

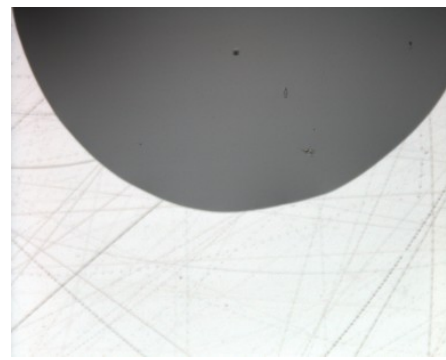

b)

Fig. 9 The petroleum drop on the glass blade in the absence (a) and under the influence of the plasma jet applied during 600s (b).

One remarks that the petroleum drop is not stretched along the surface. It was shown the plasma jet produce structural modifications of the surface [21, 22], which leads to modifications of the surface tension. However, the investigated drop preserves its spherical shape, whereas the curvature radius remains unchanged (Fig.9b). In the absence of the plasma jet treatment, the drop extends in time and produces the wetting of the surface.

\section{Conclusions}

The new constructed plasma generator, consisting in a nozzle subassembly and an electrode subassembly fixed in a common body, is a flexible device. Through the interchangeable nozzle, the plasma jet can take the shape of a needle or the shape of a cylinder, with pre-determined diameter.

The useful length of the plasma jet does not overcome $0.015 \mathrm{~m}$. It can be adjusted from the value of the tuning capacity for a fixed argon flow. The temperature of the surface, which meets the argon plasma jet, does not exceed $315 \mathrm{~K}$.

The tuning of the condenser capacity as well as of the dug diameter leads to modifications of the volume of the plasma-jet.

Due to the installation capabilities and flexibility, a large spectrum of applications can be accomplished. Figures 5-9 give eloquent examples of induced changes in several plasma treated materials. 


\section{Acknowledgements}

The authors thank Stefan Novaconi (National Institute for Research and Development in Electrochemistry and Condensed Matter Timisoara) for the manufacturing and providing the high frequency source, Professor Papp Erchardt, Professor Nicoleta Stefu and Professor Aurel Ercuta (from West University of Timisoara) for helpful discussions.

\section{References}

[1] T. J. M. Boyd, and J. J. Sanderson, "The physics of plasmas", Cambridge University Press, Cambridge (2003)

[2] I. Bica, "The Physics and the Technology of Materials in Plasma”, Mirton Press, Timisoara (2005)

[3] I. Bica, Rev. de Sold., Spain, 26 (1996) 191-200

[4] I. Bica, J. Ind. Eng. Chem., 14 (2008) 230-235

[5] I. Bica, J. Ind Eng. Chem., 15 (2009) 304-315

[6] R. Hippler, S. Pfan, and M. Schmidt, "Low temperature plasma physics: fundamental aspects and applications", Wiley VCH, Berlin (2001)

[7] J. Reece Roth, "Industrial Plasma Engineering, vol. 2; Applications to nonthermal plasma processing”, IOP Publishing Ltd, Cornwall, UK (2001)

[8] R. d'Agostino, P. Favia, Y. Kawei, H. Ikegami, N. Sato, and F. Arefi-Khonsari, “Advanced Plasma Technology”, Wiley-VCH Verlag GmbH\&Co K Cron A, Weinheim (2008)

[9] M. Thiyagarajan, I. Alexeff, S. Parameswaran, and S. Beebe, IEEE Transactions on Plasma Science, 33 (2005) 322-323

[10] J. Grabarczyk, and I. Kotela, Journal of Achievements in Materials and Manufacturing Engineering, 37 (2009) 277-280

[11] G.-Ch. Kim, H. J. Lee, and C.- H. Shon, Journal of the Koreean Physical Society 54, (2009) 628-632

[12] R. E. J. Sladek, E. Stoffels, R.Walraven, P. J. A. Tielbeek, and R. A. Koolhoren, IEEE Transactions on Plasma Science, 32 (2004) 1540-1543

[13] S. D. Anghel, I Frentin, and A. Simon, The Open Plasma Physics Journal, 2 (2009) 8-16 [14] M. Laroussi, M. G. Kong, G. Morfill, and W. Stolz Eds., „Plasma Medicine-Applications of Low-Temperature Gas Plasmas in Medicine and Biology" University Press, Cambridge (2012) 
[15] S.K. Pankaj, C. Bueno-Ferrer, N.N. Misra, V. Milosavljević, C.P. O'Donnell, P. Bourke, K.M. Keener, P.J. Cullen, Trends in Food Science \& Technology, 35(1) (2014) 5-17

[16] N. Gherbanovschi, Gh. Petrescu, "Inductive Plasma" (in Romanian), RSR Academy Press, Bucharest (1983)

[17] S. Pekárek, Acta Polytechnica, 43 (2003) 47-51

[18] P. L. F. Giangrande, Brit. J. Haematology 121, (2003) 703-712

[19] S. U. Kalghatgi, G. Fridman, M. Cooper, G. Nagaraj, M. Peddinghaus, M. Balasubramanian, V. N. Vasilets, A. F. Gutsol, A. Fridman, and G. Friedman, IEEE Transactions on Plasma Science, 35 (2007) 1559-1565

[20] R. Morent , N. De Geyter , J.Verschuren, K. De Clerck, P. Kiekens, and C. Leys, Surface \& Coatings Technology, 202 (2008) 3427-3449

[21] T. Yamamoto, M. Okubo, N. Imai, and Y. Mori, Plasma Chemistry and Plasma Processing, 24 (2004) 1-12

[22] T. Desmet, R. Morent, N. De Geyter, C. Leys, E. Schacht and P. Dubruel, Biomacromolecules, 10(9) (2009) 2351-2378 